\title{
Excimer Laser-produced Biodegradable Photopolymer Scaffolds Do Not Induce Immune Rejection In Vivo
}

\author{
Balázs FARKAS ${ }^{1, \S}$, Adam ZSEDENYI ${ }^{2, \S}$, Edina GYUKITY-SEBESTYEN ${ }^{3}$, Ilaria ROMANO ${ }^{1}$, Katalin NAGY $^{2}$, \\ Alberto DIASPRO $^{1}$, Fernando BRANDI ${ }^{1,4}$, Krisztina BUZAS $^{2,3}$ and Szabolcs BEKE ${ }^{*}$ \\ ${ }^{1}$ Department of Nanophysics, Istituto Italiano di Tecnologia (IIT), Via Morego 30, \\ 16163 Genova, Italy \\ ${ }^{2}$ University of Szeged, Faculty of Dentistry, Tisza Lajos krt. 64. H-6720 Szeged, Hungary \\ ${ }^{3}$ Hungarian Academy of Sciences, Biological Research Centre, Temesvari krt. 62, \\ H-6726 Szeged, Hungary \\ ${ }^{4}$ Istituto Nazionale di Ottica, Via Moruzzi 1, 56124-Pisa, Italy \\ *Corresponding author's e-mail: szabolcs.beke@iit.it
}

$\S$ These authors equally contributed to the work

\begin{abstract}
Following our previous works of in-vitro tests, the biocompatibility of photopolymer scaffolds was tested against immune responses in vivo. Neither relevant immune reactions nor the rejection of implanted scaffolds was detected, being an essential step for in vivo implantation of excimer laserprepared scaffolds. The scaffolds were fabricated by UV excimer laser photocuring at $308 \mathrm{~nm}$. After two weeks of transplantation neither inflammatory response nor reactive immune activation was detected based on the chemokine and cytokine profile. As a sign of biodegradability of the scaffolds, we detected macrophage infiltration and phagocytosis of the biopolymer at the site of implantation. Our results suggest that poly(propylene fumarate) $(\mathrm{PPF})$ : diethyl fumarate (DEF) $(7: 3 \mathrm{w} / \mathrm{w})$ scaffolds have appropriate properties for in vivo applications.

DOI: $10.2961 / \mathrm{jlm}$.2015.01.0002
\end{abstract}

Keywords: excimer lasers, scaffold fabrication, biocompatibility; biodegradation; in vivo experiments

\section{Introduction}

One of the major goals of tissue engineering [1-2] is to provide rapid and reliable production of well-designed and functional microenvironments called scaffolds. These scaffolds help to reestablish the structural integrity of tissues damaged by trauma, disease or aging.

Our novel Mask Projection Excimer Stereolithography (MPExSL) [3] is a versatile and fast tool to fabricate such microenvironments from liquid polymeric resins through UV photocuring. Yet, the biocompatibility [4] of these scaffolds must also be one of the primary factors considered before the structures may be used in therapeutic applications.

The validity of poly(propylene fumarate) (PPF):diethyl fumarate (DEF) $(7: 3 \mathrm{w} / \mathrm{w})$ samples fabricated by our MPExSL system has already been investigated by a previous study with bone cell culturing [5], while elastine [6-7] and Titanate nanotubes [8-9] were utilized as functional coatings. Following all these in vitro cell tests, in vivo biological testing of such PPF:DEF scaffolds became a fundamental aspect of our further progress.

\section{Materials and Methods}

\subsection{Laser photocuring and setup}

In MPExSL, schematically illustrated in Fig. 1, the image of a mask is projected on the photosensitive liquid resin [10] defining the inner geometry of the solidified polymer scaffold.

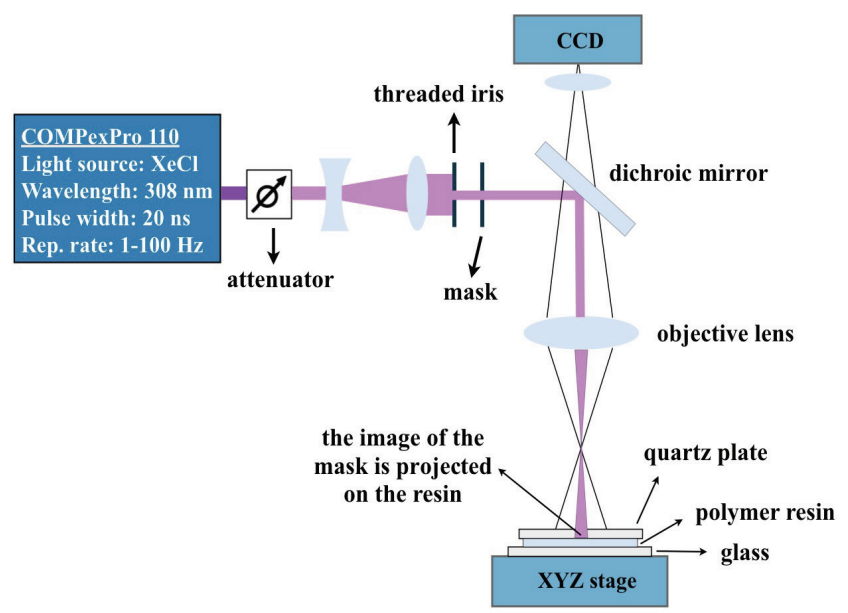

Fig. 1: MPExSL in a one-layer photocuring setup 
The resin is sandwiched between a glass holder and a quartz plate [11], in favor of a simpler and faster one-layer photocuring process. The sample is then mounted on an $\mathrm{XYZ}$ stage.

The outer geometry can be modulated by an iris. Pulse fluence of the $\mathrm{XeCl}$ excimer laser is controlled by a variable attenuator. Pulse repetition rate ranges from 1 to 100 Hz. A CCD camera is mounted on the top of the optical system to in-situ monitor the process.

The system is entirely driven by a computer. Depending on the programming, this results in different scaffold geometries (as seen on Fig. 2) [3,5].
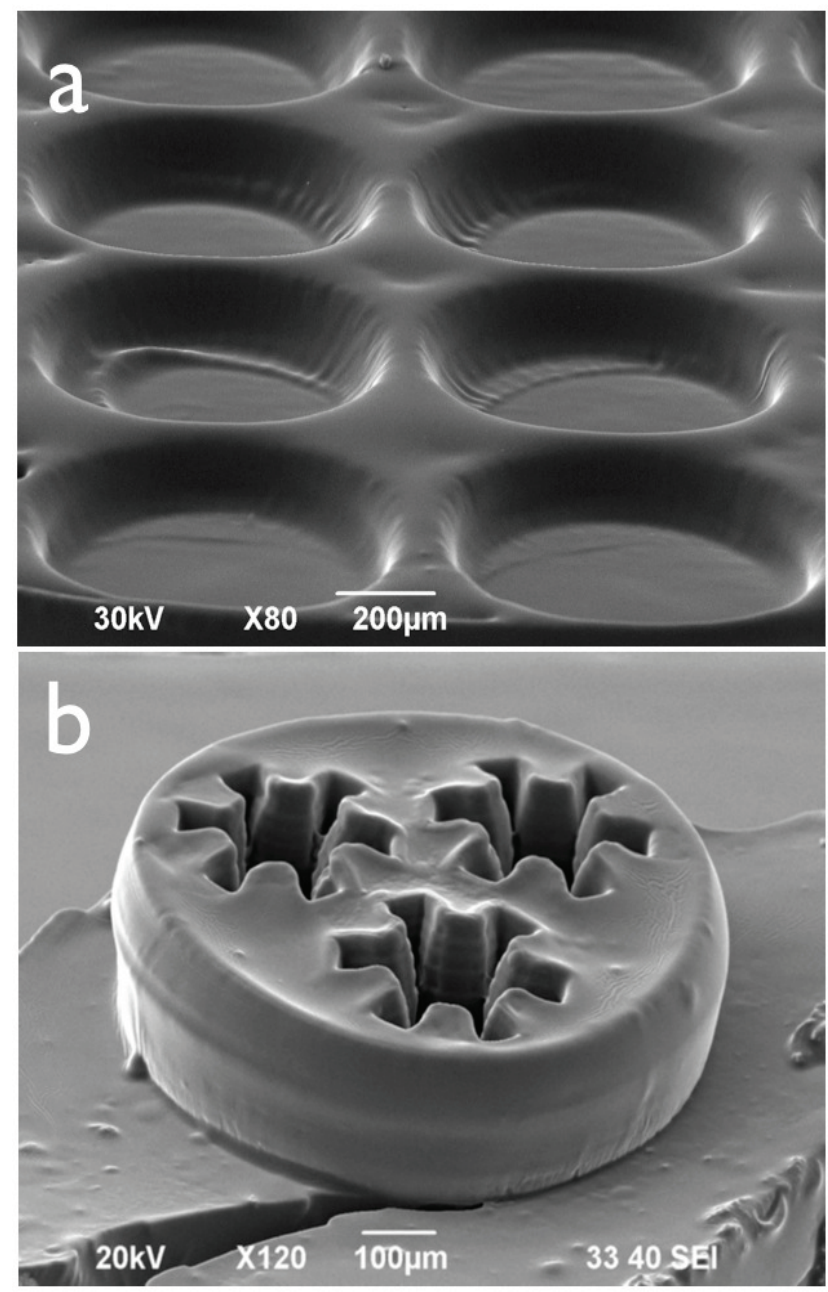

Fig. 2 (a) 5-mm diameter, 200- $\mu \mathrm{m}$ thick scaffold with pore size of $800 \mu \mathrm{m}$; (b) $2-\mathrm{mm}$ diameter, $250-\mu \mathrm{m}$ thick scaffold with cogshaped pores

\subsection{Polymer resin}

Scaffolds fabricated from PPF:DEF (7:3 w/w) [11-12] were implanted. The structures were constructed by applying UV excimer laser photocuring at $308 \mathrm{~nm}$. The photocross-linking density is tuned by photoinitiator phenylbis (2,4,6-trimethylbenzoyl) phosphine oxide (BaPO). Scaffold thickness can be adjusted by the total fluence (in this study by changing the number of shots). This tuning capability is discussed in section 3.1.
2.3 Biological testing: cell culture, mouse model, histology, and cytokine/chemokine profile

The photocured samples were sterilized with UV irradiation for $30 \mathrm{~min}$ and coated with $0.01 \%$ poly-L-lysine (MW 70000-150000 Da, Sigma) for $30 \mathrm{~min}$ at room temperature. Poly-L-lysine was removed and the scaffolds were dried under laminar box, and then incubated in Eagle's Minimum Essential Medium (EMEM, Lonza) with $10 \%$ fetal bovine serum, $100 \mathrm{U} / \mathrm{ml}$ penicillin, $100 \mathrm{U} / \mathrm{ml}$ streptomicin and $0.25 \mu \mathrm{g} / \mu \mathrm{l}$ fungizone (Promocell) for 7 days at $37^{\circ} \mathrm{C}$. The medium was changed every other day.

At day seven, K7M2 mouse cells (provided by Dennis Klinman, NCI-Frederick, MD, USA) were seeded in EMEM at $2 \times 10^{5}$ cells $/ \mathrm{ml}$ in wells containing one scaffold each, and then incubated in humidified atmosphere with $5 \% \mathrm{CO}_{2}$ at $37{ }^{\circ} \mathrm{C}$ for 14 days. After being covered with cells, the scaffolds were implanted under the dorsal skin of 8-week old female Balb/C mice (Charles River Laboratories International, Inc.). Control groups were implanted with cell-free scaffolds. 14 days after transplantation, the scaffolds and dorsal skin were removed and blood samples were collected. Five mice per group were implanted.

In case of a successful cell implantation, the presence or absence of selected K7M2 mouse osteosarcoma cell line was followed by histology. Specimens were fixed in $4 \%$ buffered paraformaldehyde and then embedded into paraffin blocks. Four- $\mu \mathrm{m}$-thick sections were prepared and stained by conventional hematoxilin-eosin stain then coverslipped. The sections were visualized by scanning virtual microscope (3D Histech, Hungary).

The potential inflammatory reactions of the implanted biomaterial were screened via cytokine and chemokine profile. Protein concentrations of the sera were measured by BCA Protein Assay (Thermo Scientific) and pooled samples were tested to simultaneously detect relative levels of different cytokines according to the manufacture's instruction by Mouse Cytokine Array, Panel A (R\&D Systems). Immunoreactive signals were detected using a LICOR ODYSSEY ${ }^{\circledR}$ Fc (Dual-mode imaging system) imager followed by analysis with Odyssey v1.2 software. Animal care was provided in accordance with the procedures outlined in the animal protocol authorized by the Institutional and the National Animal Ethics and Experimentation Boards.

\section{Results and discussion}

\subsection{Preliminary results for PPF:DEF (7:3 w/w) scaffold fabrication}

The wavelength of a XeCL excimer laser (308 nm) has the greatest penetration depth of all excimer sources in PPF:DEF and thus highly desirable for the scaffold fabrication process [11]. Apart from the wavelength of the laser, the thickness of the polymerized layer can also be tuned by other laser parameters [13], such as the applied total fluence. The range of this tuning is strongly dependent on the resin's photoinitiator concentration. Thus, the layer thickness dependence over the $\mathrm{BaPO}$ (Fig. 3) was acquired for PPF:DEF $(7: 3 \mathrm{w} / \mathrm{w})$ to be able to achieve the proper layer thickness of any desired structure.

For all measurements, non-porous layers were fabricated with different $\mathrm{BaPO}$ concentrations using a repetition 
rate of $50 \mathrm{~Hz}$ and fluence per pulse of $20 \mathrm{~mJ} / \mathrm{cm}^{2}$. The applied numbers of pulses were 50, 124, 248, 336, 480, 720 and 1000. Height measurements were performed with a Veeco Dektak 150 profiler. BaPO concentration ranged from $0.3 \%$ to $1.2 \%$. We assume that the observed sublinear dependence is due to the $308 \mathrm{~nm}$ light having greater penetration depth in the crosslinked resin than in the pristine one.

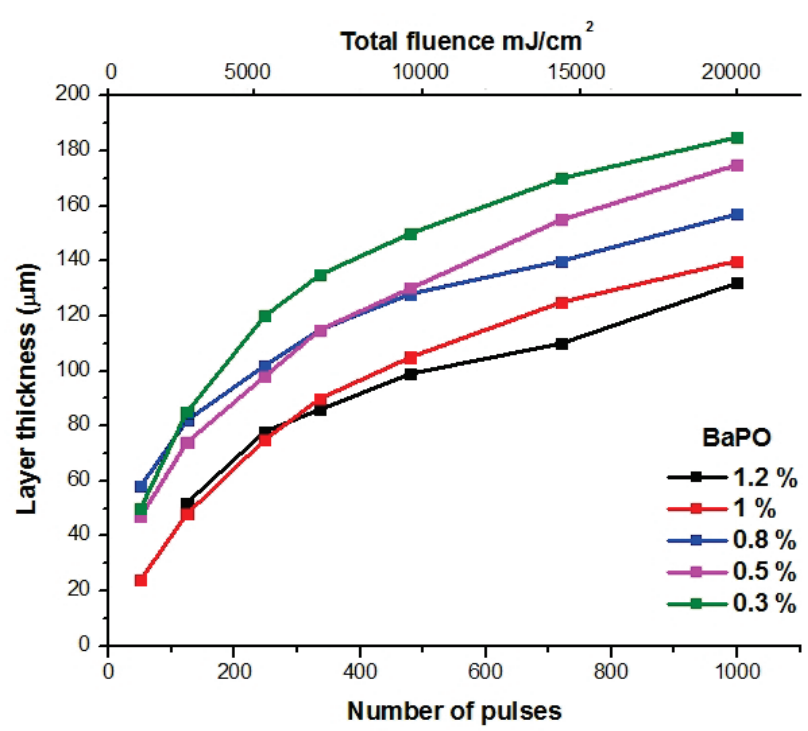

Fig. 3: Layer thickness as a function of number of pulses (and total fluence) in case of various $\mathrm{BaPO}$ concentrations

For the in vivo tests, $5-\mathrm{mm}$ diameter, $100-\mu \mathrm{m}$ thick porous scaffolds were prepared with $55-\mu \mathrm{m}$-pore diameters, using a BaPO concentration of $1 \%$. These have been presented in [9].

\subsection{In-vivo biocompatibility of monolayer scaffolds}

K7M2 mouse osteosarcoma cell line was grown on the scaffolds for 14 days before implantation in mice. The control group was implanted with cell-free scaffolds. After 14 days, the scaffolds and dorsal skin were removed and serum samples were harvested for further analysis.

Cytokine and chemokine protein levels in sera were also investigated. No significant difference was detected between the cytokine profiles of the tumor scaffold implanted group and the control group (Fig 4). No sign of inflammation was observed on the dorsal skin: neither inflammatory cytokines, nor other signs of rejective reaction were found.

In histological sections (Fig. 5) of the group implanted with tumor seeded scaffolds (b), viable anaplastic sarcomatous tumor cells embedded in dens inflammatory reaction were seen (d-interrupted line indicates tumor border), in contrast to the control group (a) where only a mild fibrotic reaction was noted around the presumable location of the scaffold (Fig 8c-arrows).

No residual biopolymer piece was found in the control animals. We presume that the scaffolds degraded biologically in vivo without significant residual pieces.
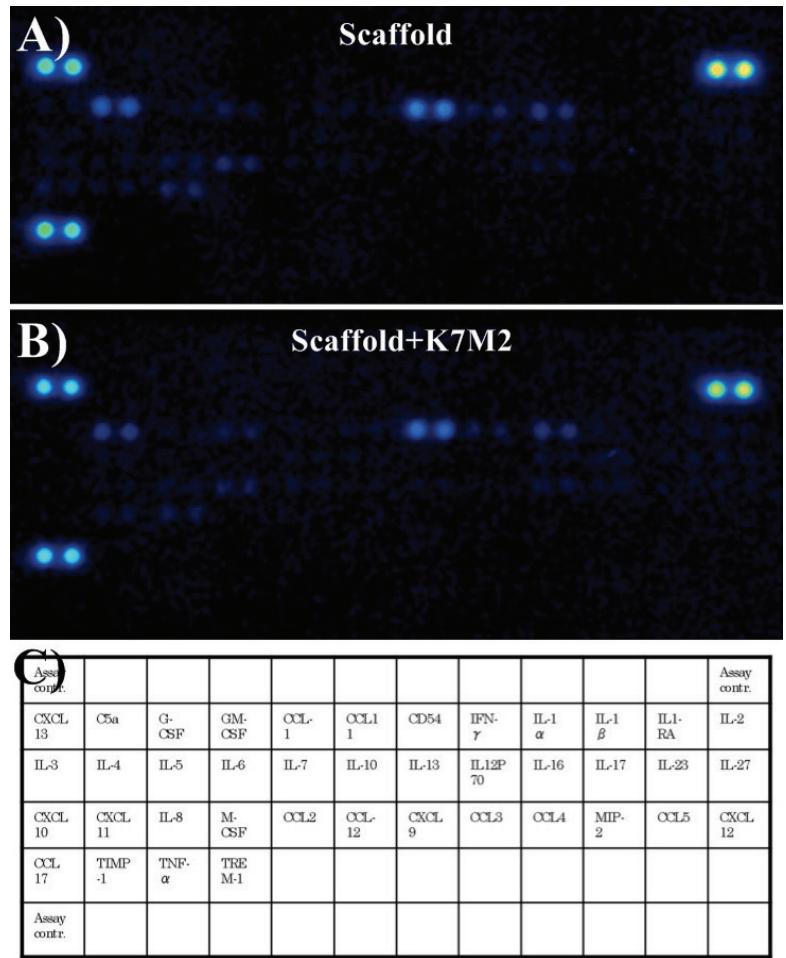

Fig. 4: profiling of (A) tumor scaffold implanted mice sera and (B) control groups sera. Panel (C) shows the cytokine and chemokine map



Fig. 5: HE; OM 200x; a-b and c-d scale bar $1000 \mu \mathrm{m}$ and $200 \mu \mathrm{m}$, respectively

\section{Conclusions}

PPF:DEF scaffolds were fabricated by our excimer laser stereolithography system and have been tested in vivo with K7M2 mouse osteosarcoma cell line. No inflammatory reaction or other rejection was observed opening up the possibility of utilizing the aforementioned scaffolds in biological applications, while the MPExSL presents a fast, versatile, and reliable way to fabricate simple photocured structures for tissue engineering and regenerative medicine.

\section{Acknowledgments and Appendixes}

This work has received funding support from the European Union (FP7-NMP-2013-EU-China) grant agreement n.604263 (NEUROSCAFFOLDS) and the grant of The Hungarian Social Renewal Operational Program (TAMOP4.2.2-A-11/1/KONV-2012-0025). The project is cofinanced by the European Union and the European Social Fund. 


\section{References}

[1] R. Langer and J. P. Vacanti, „,Tissue engineering”, Science 260, 920-926 (1993)

[2] Tabata, Y., „Biomaterial technology for tissue engineering applications", J. R. Soc. Interface 6, S311S324 (2009)

[3] S. Beke, B. Farkas, I. Romano, F. Brandi, , 3D scaffold fabrication by Mask Projection Excimer laser Stereolithography", Vol. 4, Issue 10, pp. 2032-2041 (2014), http://dx.doi.org/10.1364/OME.4.002032

[4] Williams, D. F., ,The Williams dictionary of biomaterials", Liverpool, UK: University Press (1999)

[5] S Beke, F Anjum, H Tsushima, L Ceseracciu, E Chieregatti, A Diaspro, A. Athanassiou, F. Brandi, „Towards excimer-laser-based stereolithography: a rapid process to fabricate rigid biodegradable photopolymer scaffolds", Journal of The Royal Society In$\begin{array}{llll}\text { terface } & 9 & \text { (76), } & 3017-3026 \quad \text { (2012), }\end{array}$ http://dx.doi.org/10.1098/rsif.2012.0300

[6] S Scaglione, R Barenghi, S Beke, L Ceseracciu, I Romano, F Sbrana, P. Stagnaro, F. Brandi, M. Vassalli, "Characterization of bioinspired elastin-polypropylene fumerate material for vascular prostheses applications", SPIE Optical Metrology, 87920H-87920H-6 (2013) http://dx.doi.org/10.1117/12.2021754

[7] R. Barenghi, S. Beke, I. Romano, P. Gavazzo, F. Sbrana, M. Vassalli, F. Brandi, S. Scaglione „Elastin-coated biodegradable photopolymer scaffolds for enhanced cell adhesion and proliferation" BioMed Research International, vol. 2014, Article ID 624645, 9 pages, (2014). http://dx.doi.org/10.1155/2014/624645
[8] S Beke, L Körösi, A Scarpellini, F Anjum, F Brandi, „Titanate nanotube coatings on biodegradable photopolymer scaffolds", Materials Science and Engineering: C 33, Pages 2460-2463 (2013), http://dx.doi.org/10.1016/j.msec.2013.01.066

[9] S. Beke, R. Barenghi, B. Farkas, I. Romano, L. Körösi, S. Scaglione, F. Brandi, ,Improved cell activity on biodegradable photopolymer scaffolds using titanate nanotube coatings" Materials Science and Engineering C, 44, 38-43, (2014), http://dx.doi.org/10.1016/j.msec.2014.07.008

[10] Ha, Y. M., Choi, J. W. \& Lee, S. H. „Mass production of 3-D microstructures using projection microstereolithography", J. Mech. Sci. Technol. 22, 514-521 (2008)

[11] S Beke, F Anjum, L Ceseracciu, I Romano, A Athanassiou, A Diaspro, F Brandi, „Rapid fabrication of rigid biodegradable scaffolds by excimer laser mask projection technique: a comparison between 248 and 308 nm", Laser Physics 23 (3), 035602, (2013), doi:10.1088/1054-660X/23/3/035602

[12] Kasper, K. F., Tanahashi, K., Fisher, J. P. \& Mikos, A. G., ,Synthesis of poly(propylene fumarate)”, Nat. Protocol 4, 518-525. (2009)

[13] B. Farkas, I. Romano, L. Ceseracciu, A. Diaspro, F. Brandi, S. Beke, "Four-order stiffness variation of laser-fabricated photopolymer biodegradable scaffolds by laser parameters modulation", Materials Science and Engineering: C, 2015

(Received:July 18, 2014, Accepted: November 23, 2014) 